Case Report

\title{
Intestinal Necrosis Due to Digoxin Intoxication
}

Line L. Olesen *

Department of Cardiology, Zealand University Hospital (Roskilde), Sygehusvej 10, 4000 Roskilde, Denmark; E-Mail: llole@regionsjaelland.dk

* Correspondence: Line L. Olesen; E-Mail: Ilole@regionsjaelland.dk

Academic Editor: Bodh I. Jugdutt

Special Issue: Aging and Heart Failure

OBM Geriatrics

2020, volume 4 , issue 2

doi:10.21926/obm.geriatr.2002115
Received: February 01, 2020

Accepted: April 15, 2020

Published: April 21, 2020

\begin{abstract}
The knowledge about digoxin, the associated cardiac and extracardiac effects, its pharmacokinetics and dynamics, indications, dosing, and side effects is important with the objective of deriving its maximum benefit without the risk of intoxication. Although the dosing of digoxin has been standardized and its usage has declined, digoxin intoxication still occurs frequently. Due to the risk of fatal complications, it has a negative prognosis. The knowledge about the etiology and symptoms of intoxication is critical for the correct diagnosis and treatment of patients, to prevent lethal complications, and to improve prognosis. A case of lethal extensive intestinal necrosis caused by digoxin intoxication is reported. An early recognition of this rare, unusual, and serious complication should prompt life-saving treatment. Prevention is advocated by treating with activated charcoal or cholestyramine to reduce digoxin absorption, potassium (S-K $5 \mathrm{mmol} / \mathrm{L}$ ) to reduce digoxin toxicity, digoxin antibody, Digibind, an antidote used to neutralize digoxin, and vasodilatation against digoxin induced vasoconstriction.
\end{abstract}

\section{Keywords}

Intestinal necrosis; vasoconstriction; digoxin intoxication; prevention and cure; case reports

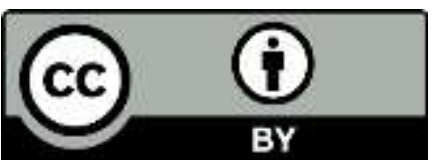

(C) 2020 by the author. This is an open access article distributed under the conditions of the Creative Commons by Attribution License, which permits unrestricted use, distribution, and reproduction in any medium or format, provided the original work is correctly cited. 


\section{Introduction}

\subsection{The History of Digitalis and the Cardiac Glycosides [1]}

Several centuries back, a physician named William Withering (1741-99) became famous for discovering that the foxglove plant (Digitalis, Figure 1) contains various cardioactive steroids/glycosides (digitoxin, digoxin, ouabain). These have a diuretic effect and are beneficial for patients with congestive heart failure. They are also helpful in slowing down the pulse and are beneficial in patients with atrial flutter and fibrillation. In those days there was only little treatment to offer patients with such cardiac problems.

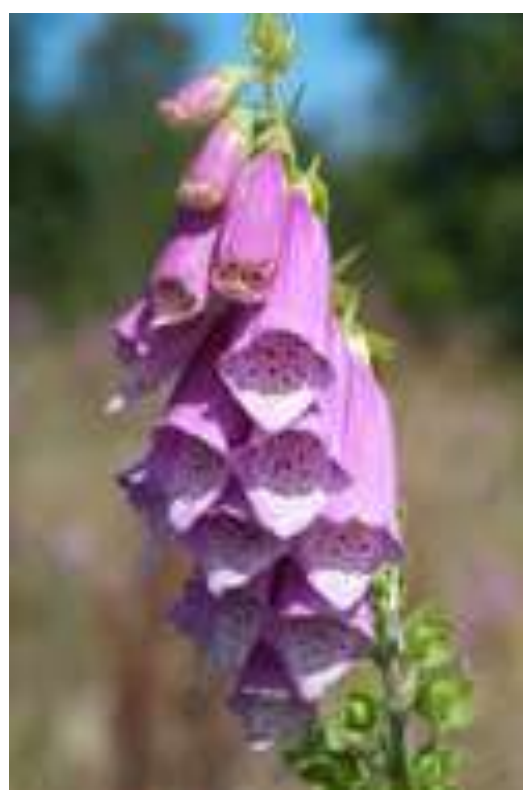

Figure 1 Digitalis.

The patients were administered powdered leaves from the foxglove plant, which contain variable amounts of glucosides, mostly digitoxin. Consequently, the dosage was determined by the clinical effect and mainly by the presence of side effects on the gastrointestinal tract or other signs of digitalis intoxication. Digitalis intoxication was a major problem in those days and made it unpopular.

Treatment with powdered foxglove continued to the 19th century when it became possible to produce standardized digitalis tablets. Digoxin has become the most widely used glycoside all over the world during the last 30 years as it is preferred over digitoxin due to its shorter half-life. Concurrently, medicines far more efficient have been developed, limiting the indications for treatment with digoxin.

Still, digoxin is a mainstay in medicine, and digoxin intoxication remains a challenge.

\subsection{Intestinal Necrosis Due to Digoxin Intoxication}

In 1961, PC Gazes et al. were the first to describe cases of lethal digoxin intoxication complicated by intestinal necrosis [2]. A number of publications and experimental studies revealed that ouabain could induce splanchnic vasoconstriction [3-5]. However, this complication has been almost forgotten and presumably overlooked. 
In 1978, when I was a young student working in a department of pathology, I performed an autopsy of a patient who had died from digitalis intoxication and found that the intestines were black, hemorrhagic, and gangrenous. The aging assistant remembered that he had observed similar findings earlier in patients with digitalis intoxication. I subsequently trained to become a cardiologist and now, forty years later, discovered another case of lethal extensive intestinal necrosis in a patient with digoxin intoxication.

\section{Case Presentation}

A 73-year old male was admitted to the hospital with the complaint of dizziness and weakness following long-term anorexia and weight loss.

He had a history of (1) chronic atrial fibrillation, which was rate-regulated with digoxin $0.25 \mathrm{mg}$ and selozok $200 \mathrm{mg}$ daily, (2) arterial hypertension, treated with Corodil $40 \mathrm{mg}$ daily, (3) liver cirrhosis, ascites, and esophageal varices, treated with spironolactone $100 \mathrm{mg}$ daily, and (4) normal biochemistry and a BMI of 30. EKG showed typical digoxin changes one month before admission (Figure 2).

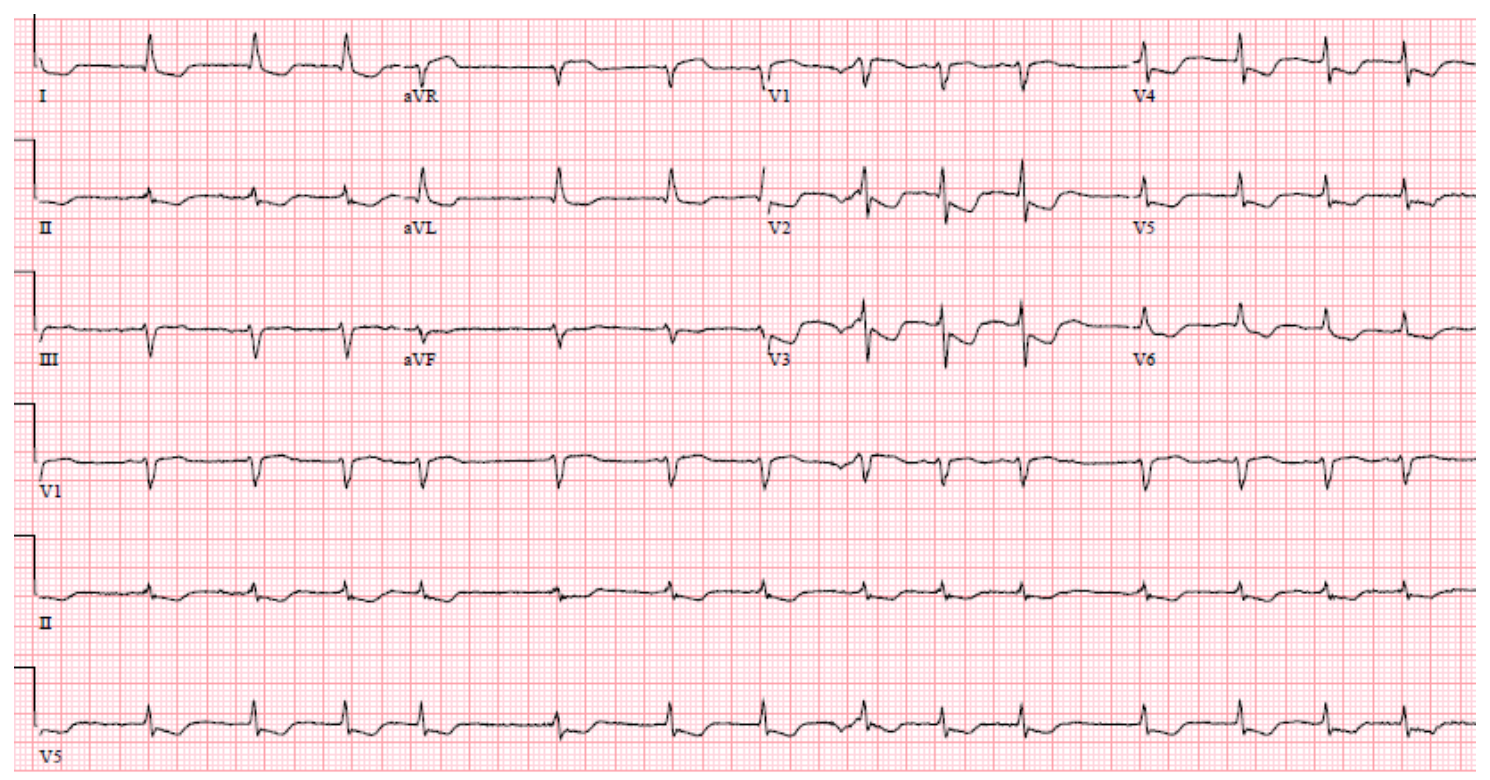

Figure 2 EKG with typical digoxin changes: Short QT-interval with secondary repolarization abnormalities affecting the ST-segments, T-waves, and U-waves.

On admission, he was dehydrated, hypotensive, cold, cyanotic, and confused. EKG showed atrial fibrillation with third-degree AV-block and broad escape rhythm, 30 beats/min (Figure 3).

Serum digoxin concentration (S-digoxin) > 6, $4 \mathrm{nmol} / \mathrm{L}$, GFR 7, S-creatinine $654 \mathrm{mmol} / \mathrm{L}, \mathrm{S}-\mathrm{K} \mathrm{5,4}$ $\mathrm{mmol} / \mathrm{L}$, INR 3,8. BMI 29. Echocardiography was normal.

All the medications were withdrawn, and fluid therapy was administered. A temporary pacemaker stabilized the pulse at $70 \mathrm{bpm}$ and the blood pressure at 150/80 $\mathrm{mmHg}$. There was marked clinical improvement, and thus, it was decided that the administration of Digibind was not necessary. 


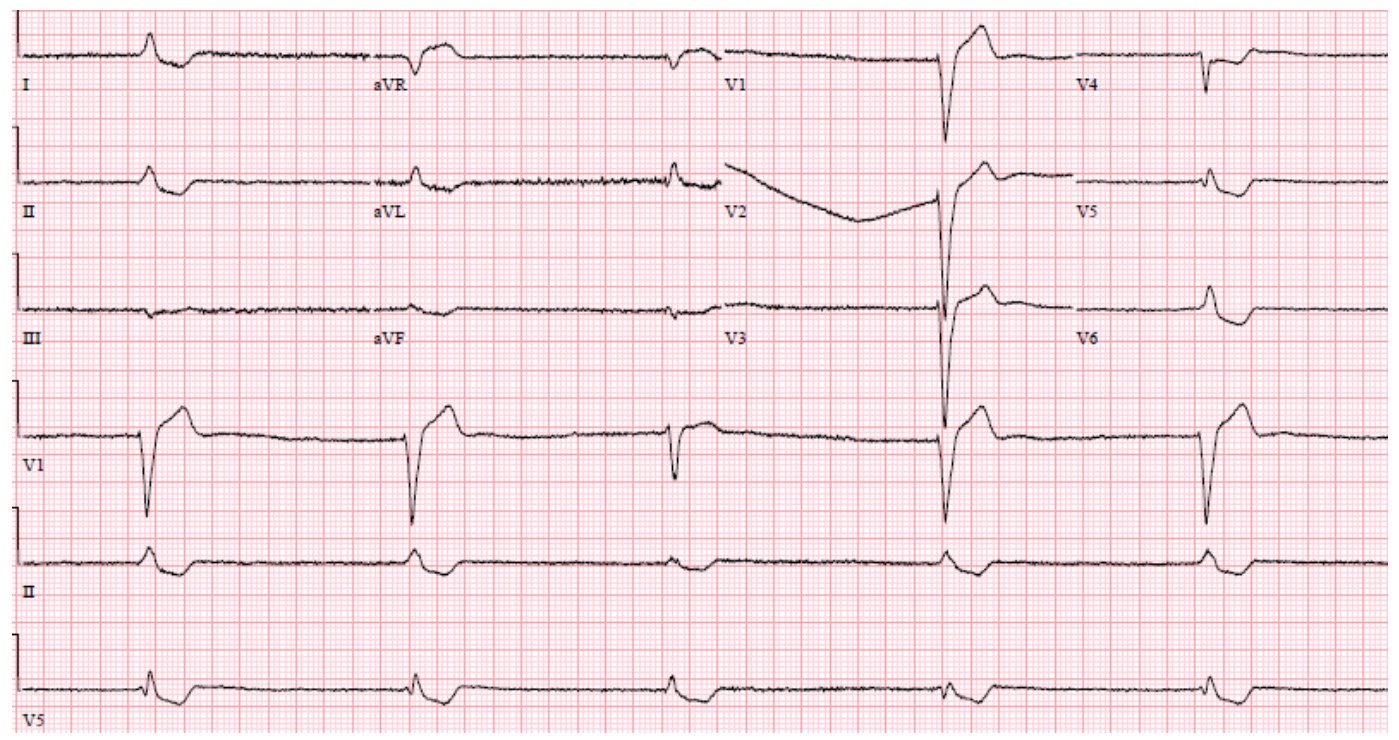

Figure 3 EKG with third degree atrioventricular block.

One and a half days later, he started to vomit and exhibited dark-colored liquid stool and pain in the abdomen, which was initially out of proportion with the objective findings. His abdomen distended, and bowel sounds disappeared. He was uremic with S-digoxin $>6,4 \mathrm{nmol} / \mathrm{L}$ and S-K 4 $\mathrm{mmol} / \mathrm{L}$. S-lactate increased from 1,5 to $4,8 \mathrm{mmol} / \mathrm{L}$. He had a normal temperature, leukocyte count, and CRP, but Enterobacter cloacae were detected in the blood.

CT of the abdomen showed pneumatosis (gas cysts) in the walls of the distal esophagus, the ventricle and duodenum (Figure 4), and the small intestine (Figure 5), corresponding to intestinal ischemia and necrosis. Moreover, there was air in the superior mesenteric vein and other mesenteric veins and free air in the abdomen. Aorta was severely atherosclerotic, but not stenotic. No treatment was possible, and he died a day later, i.e., two and a half days after admission. No autopsy was performed.

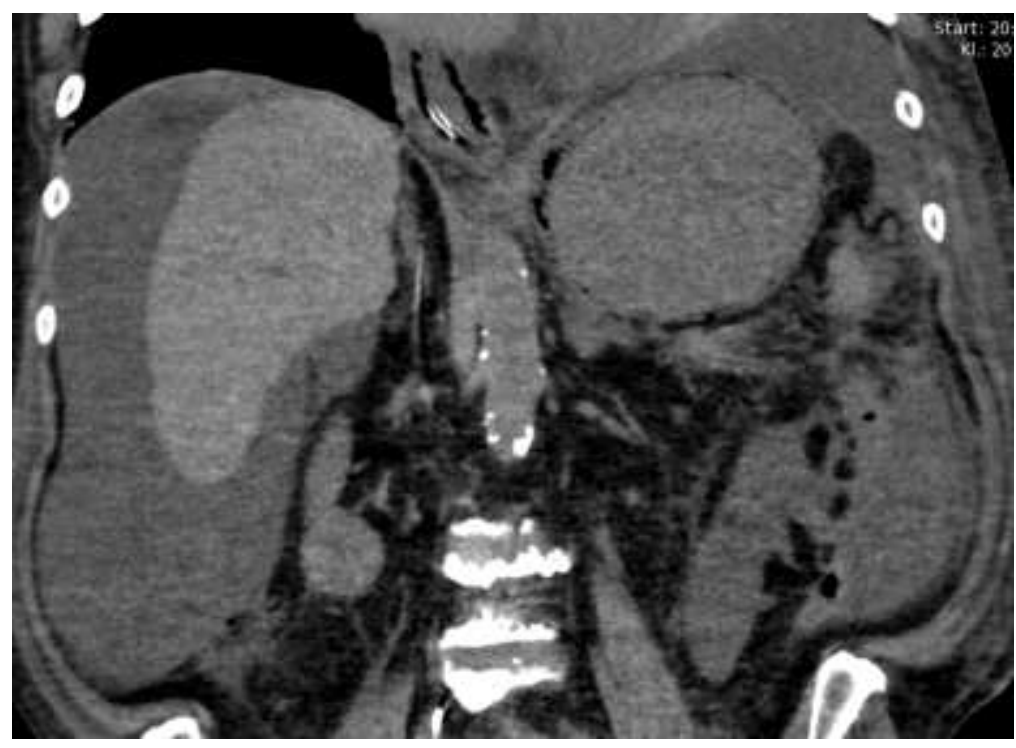

Figure $4 \mathrm{CT}$ of the abdomen with pneumatosis in the wall of the distal part of the esophagus and the ventricle. 


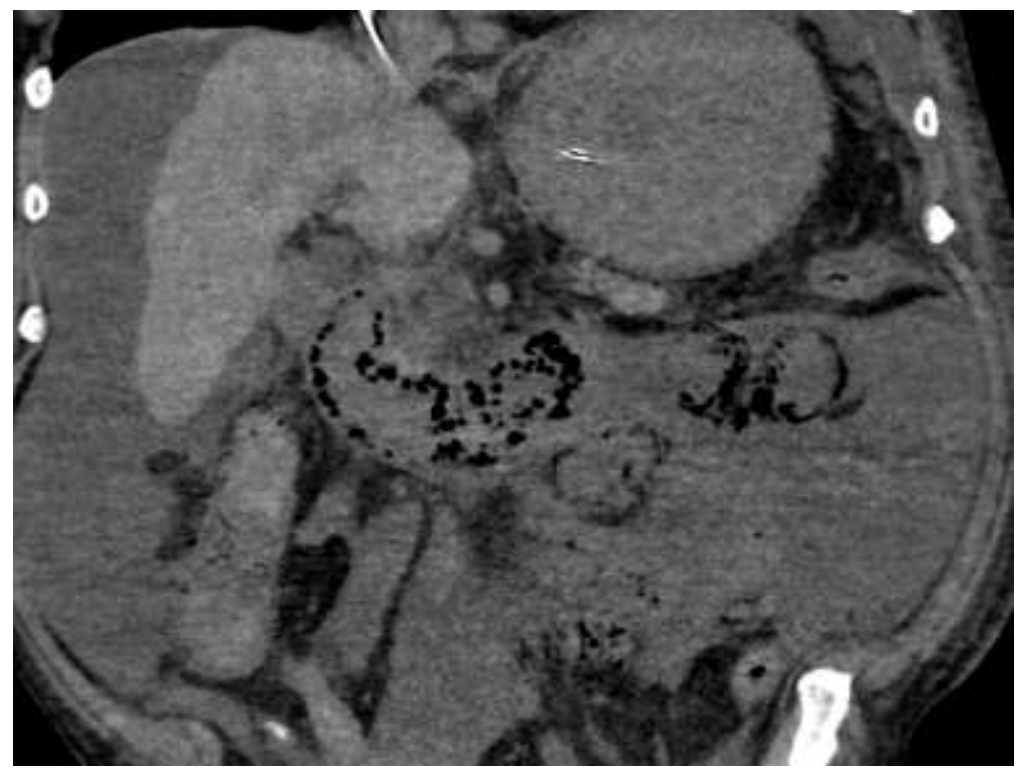

Figure $5 \mathrm{CT}$ of the abdomen with pneumatosis in the small bowel wall.

\section{Discussion}

The case report suggests long-lasting side effects of digoxin, without a reconsideration of the indication, dosage, and measurement of S-digoxin.

Even though circulation was reestablished and all the medication was discontinued, the Sdigoxin level remained very high, indicating its ongoing absorption from the intestines and release from the tissues, in addition to decreased renal elimination, causing late intestinal ischemia. The patient did not receive treatment with either oral or intravenous digoxin-binding medicine.

Due to the prolonged and severe digoxin intoxication, he was exposed to protracted digoxininduced vasoconstriction and developed the typical symptoms of necrotizing enteritis. CT angiography, the most important diagnostic method, revealed pronounced changes in the upper part of the gastrointestinal tract. Pneumatosis (gas cysts) is a pathognomic sign of bowel gangrene and terminal intestinal disease. The free air in the abdomen is consistent with a leaky bowel, peritonitis, and high mortality. There was no treatment to offer when intestinal necrosis was finally recognized.

Stenosis of the mesenteric vessels increases the risk of under perfusion and intestinal ischemia related to circulatory failure, low-flow, dehydration, bradycardia, hypotension, and compensatory vasoconstriction caused by diverse disorders, medications, and medicine intoxication [6-8]. Hence, intestinal ischemia may also be an indirect result of digoxin intoxication. However, it is more likely a direct consequence of digoxin-induced vasoconstriction, as observed in this case, which can give rise to non-occlusive mesenteric ischemia (NOMI) and should be treated with vasodilation $[2-5,8$, 9]. Allergic reaction to digoxin is unusual and, in this case, was an unlikely cause of the vascular reaction.

The indications for digoxin are to regulate the resting rate of atrial fibrillation and to treat patients with symptomatic systolic heart failure, despite optimal standard treatment. These cardiac conditions are especially frequent in the old and elderly [10].

Digoxin has a positive inotropic effect, and negative dromotropic, and chronotropic effects through inhibition of $\mathrm{Na}+\mathrm{K}+\mathrm{ATPase}$ in the cell membrane and increased vagal tone [10]. Digoxin 
decreases the duration of the action potential, shortens the refractory period, and prolongs the AV-conduction. Electrocardiographic manifestations of the electrophysiologic effects are characterized by ST-segment depression, T-wave inversion, a shortened QT-interval, and prolongation of the PR-interval (Figure 2) [11, 12].

About $70 \%$ of digoxin is absorbed by the intestines and slowly distributed in a large volume and $25 \%$ binds to protein. Digoxin is almost completely excreted in the urine in unchanged form. In patients with normal renal function, $t 1 / 2$ is $1.5-2$ days, and steady-state is $5-7$ days after the start of treatment without a loading dose. When there is complete anuria, $t 12$ is 5 days and the elimination of digoxin is mainly hepatic [10].

Digoxin has a narrow therapeutic index. It has many adverse side effects, mostly affecting the old and elderly patients, and it has several interactions. Spironolactone, amiodarone, verapamil, macrolides, and many more increase S-digoxin. Interaction should be anticipated, and the digoxin dose should be reduced to half of the original dosage to maintain the same serum concentration $[10,13]$. In patients with systolic heart failure and sinus rhythm, the positive effect of digoxin is obtained with a small dosage of 0.0625-0.125 mg.

Inappropriately high dosage is the typical cause of digoxin intoxication [10, 14]. With increasing age, there is a decrease in renal function, and it is important to reduce the digoxin dosage as the patient grows older [13]. It is advisable to monitor the stable patient annually [15], and more frequently when anything changes in the patient's condition, weight, comorbidities, and cotreatments. Digoxin dosage should be adjusted and always kept at the lowest dosage possible $[10,15]$.

Digoxin intoxication [16] is associated with arrhythmias such as ventricular fibrillation, ventricular tachycardia, asystole, atrioventricular block (first, second and third (Figure 3)), nodal rhythm, and atrial tachycardia, and with confusion, visual disturbances, nausea, vomiting, weight loss, and abdominal pain as was observed in the present case.

Treatment of digoxin intoxication is obligate, symptomatic, and preventive [17]. Besides giving standard treatment against digoxin intoxication [10], every patient should be closely monitored for symptoms, heart frequency, blood pressure, electrolytes, and acid-base status and should be treated with fluid, oxygen, and perhaps antibiotics. It should be noted that dialysis does not eliminate digoxin.

Activated charcoal $50 \mathrm{~g}$ and/or cholestyramine $4 \mathrm{~g}$, repeated after four hours, bind digoxin, reduce intestinal absorption, and increase the excretion of digoxin. In severe digoxin intoxication, it should be administered repeatedly $[17,18]$. In the event of acute intoxication, the ventricle should also be emptied.

Since hypokalemia augments digoxin toxicity, S-K close to $5 \mathrm{mmol} / \mathrm{L}$ should be achieved by administering an intravenous infusion of $10-40 \mathrm{mmol}$ potassium hourly. Precaution should be administered as hyperkaliemia may aggravate atrioventricular block. Hypomagnesaemia exacerbates digoxin toxicity and should be corrected. Hypocalcemia is beneficial, whereas calcium administration may be dangerous $[10,16]$.

Treat bradycardia with atropine and pacemaker.

Treat tachycardia with beta-blocker, ventricular tachycardia with lidocaine. Avoid cardioversion, because it can provoke intractable arrhythmias [16].

Indications for the antidote anti-digoxin antibody Fab-fragment Digibind are [14]:

Acute ingestion of $>10 \mathrm{mg}$ in adults and $>4 \mathrm{mg}$ in children 
S-digoxin $>5(-10) \mathrm{nmol} / \mathrm{L}$ (measured $6-8 \mathrm{~h}$ after ingestion)

$\mathrm{S}-\mathrm{K}>5-5,5 \mathrm{mmol} / \mathrm{L}$ (with no other explanation than digoxin intoxication)

Life-threatening symptoms (circulatory failure, abdominal pains, and intestinal ischemia) [19]

Administer a Digibind infusion of $40-80 \mathrm{mg}$ in normal saline over a span of $20 \mathrm{~min}$. The subsequent measurement of S-digoxin is futile. The treatment should be repeated after $60 \mathrm{~min}$ until the serious symptoms disappear. Digibind strongly binds and inactivates digoxin and releases it from $\mathrm{Na}+\mathrm{K}+$ ATPase. The maximum effect is obtained after $90 \mathrm{~min}$ [14]. The indication for Digibind is enhanced when the elimination of digoxin is limited due to renal failure.

Consider treating vasoconstriction with vasodilation using glucagon, papaverine, calciumblockers, which are papaverine derivatives, and/or nitrates, especially in case of severe digoxin intoxication combined with abdominal pains and/or known stenosis in mesenteric vessels to prevent or cure intestinal ischemia [7-10, 20].

\section{Acknowledgment}

I want to thank the Department of Radiology, Roskilde, Denmark, for consent to use Figure 4 and Figure 5.

\section{Author Contributions}

Line L. Olesen did all the work.

\section{Competing Interests}

The author has declared that no competing interests exist.

\section{References}

1. Nielsen BL. Heart diseases and treatment of heart failure. Cardiologisk Forum. 1998; 3: 26-34.

2. Gazes PC, Holmes CR, Moseley V, Pratt-Thomas HR. Acute hemorrhage and necrosis of the intestines associated with digitalization. Circulation. 1961; 23: 358-364.

3. Ferrer MI, Bradley SE, Wheeler HO, Enson Y, Preisig R, Harvey RJM. The effect of digoxin in the splanchnic circulation in ventricular failure. Circulation. 1965; 32: 524-537.

4. Cohen SE, Feldman MI, Wolfman Jr EF. Nonocclusive hemorrhagic necrosis of the intestine. West J Med. 1974; 121: 449-455.

5. Levinsky RA, Lewis RM, Bynum TE, Hanley H. Digoxin induced intestinal vasoconstriction. The effects of proximal arterial stenosis and glucagon administration. Circulation. 1975; 52: 130136.

6. Olesen L. Intestinal necrosis due to beta-blocker intoxication. Cardiologist. 2020; 1: CARDIOL01-1006.

7. Florim S, Almeida A, Rocha D, Portugal P. Acute mesenteric ischaemia: A pictorial review. Insights Imaging. 2018; 9: 673-682.

8. Oldenburg WA, Lau LL, Rodenberg TJ, Edmonds HJ, Burger CD. Acute mesenteric ischemia: A clinical review. Arch Intern Med. 2004; 164: 1054-1062.

9. Weil J, Gupta RS, Herfarth H. Nonocclusive mesenteric ischemia induced by digitalis. Int J Colorectal Dis. 2004; 19: 277-280. 
10. Whayne TF. Clinical use of digitalis: A state of the art review. Am J Cardiovasc Drugs. 2018; 18 : 427-440.

11. Cohn AE, Fraser FR, Jamieson RA. The influence of digitalis on the $T$ wave of the human electrocardiogram. J Exp Med. 1915; 21: 593-603.

12. Tiliakos M. The effect of digitalis on the normal electrocardiogram. Br Heart J. 1953; 15: 95100.

13. Kirilmaz B, Saygi S, Gungor H, Turk UO, Alioğlu E, Akyuz S, et al. Digoxin intoxication: An old enemy in modern era. J Geriatr Cardiol. 2012; 9: 237-242.

14. Pincus M. Management of digoxin toxicity. Aust Prescr. 2016; 39: 18-20.

15. Adams Jr KF, Butler J, Patterson JH, Gattis Stough W, Bauman JL, van Veldhuisen DJ, et al. Dose response characterization of the association of serum digoxin concentration with mortality outcomes in the Digitalis Investigation Group trial. Eur J Heart Fail. 2016; 18: 10721081.

16. Chung EK. Digitalis intoxication. Postgrad Med J. 1972; 48: 163-179.

17. Senior DF, Feist EH, Stuart LB, Lombard CW. Treatment of acute digoxin toxicosis with digoxin immune fab (Ovine). J Vet Intern Med. 1991; 5: 302-303.

18. Henderson RP, Solomon CP. Use of cholestyramine in the treatment of digoxin intoxication. Arch Intern Med. 1988; 148: 745-746.

19. Guglielminotti J, Tremey B, Maury E, Alzieu M, Offenstadt G. Fatal non-occlusive mesenteric infarction following digoxin intoxication. Intensive Care Med. 2000; 26: 829.

20. Brandt $\amalg$, Boley SJ. AGA technical review on intestinal ischemia. Gastroenterology. 2000; 118: 954-968.

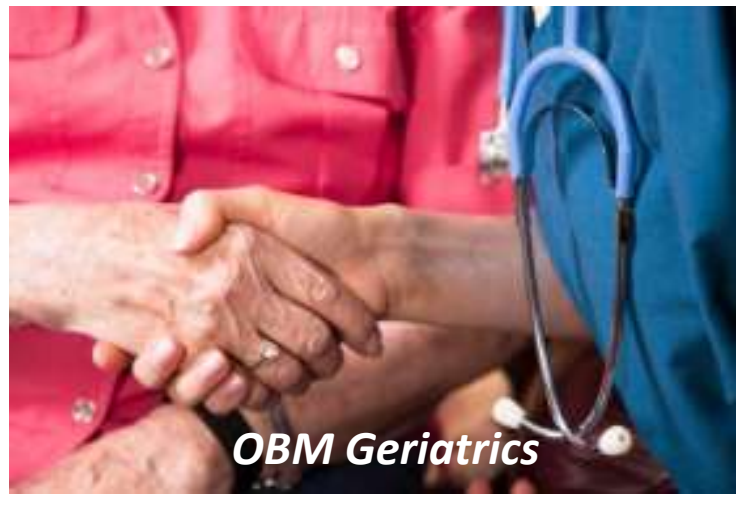

Enjoy OBM Geriatrics by:

1. Submitting a manuscript

2. Joining in volunteer reviewer bank

3. Joining Editorial Board

4. Guest editing a special issue

For more details, please visit:

http://www.lidsen.com/journals/geriatrics 